\title{
A modular invariance on the theta functions defined on vertex operator algebras
}

\author{
Masahiko Miyamoto * \\ Institute of Mathematics \\ University of Tsukuba \\ Tsukuba 305, Japan
}

\begin{abstract}
In this paper, we introduce theta-functions of VOA-modules and show that the space spanned by them has a modular invariance property. If a VOA is a lattice VOA $V_{L}$ associated with an even lattice $L$ (cf. [FLM]), then the above theta functions coincide to the ordinary theta functions $\theta(\tau, \vec{v})=\sum_{m \in L+\beta} e^{\pi \imath<m, m>\tau+<m, \vec{v}>}$ for $<\beta, L>\subseteq \mathbb{Z}$, $\vec{v} \in \mathbb{C} L$ and $\tau \in \mathcal{H}$.
\end{abstract}

\section{Introduction}

Throughout this paper, $V$ denotes a vertex operator algebra $\left(\oplus_{n=0}^{\infty} V_{n}, Y, \mathbf{1}, \omega\right)$ with central charge $c$ and $Y(v, z)=\sum v(n) z^{-n-1}$ denotes a vertex operator of $v$. (Abusing the notation, we will also use it for vertex operators of $v$ for $V$-modules.) $o(v)$ denotes the grade-keeping operator of $v$, which is given by $v(m-1)$ for $v \in V_{m}$ and defined by extending it for all elements of $V$ linearly. In particular, $o(\omega)$ equals to $L(0)=\omega(1)$ for Virasoro element $\omega$ of $V$ and $o(v)=v(0)$ for $v \in V_{1}$.

In order to simplify the situation, we assume that $\operatorname{dim} V_{0}=1$ so that there is a constant $\langle v, u\rangle \in \mathbb{C}$ such that $v_{1} u=-\langle v, u\rangle \mathbf{1}$ for $v, u \in V_{1}$.

We call $V$ a rational vertex operator algebra in case each $V$-module is a direct sum of simple modules. Define $C_{2}(V)$ to be the subspace of $V$ spanned by elements $u(-2) v$ for $u, v \in V$. We say that $V$ satisfies Condition $C_{2}$ if $C_{2}(V)$ has finite codimension in $V$. For a $V$-module $M$ with grading $M=\oplus M_{m}$, we define the formal character as

$$
\operatorname{ch}_{q} M=q^{-c / 24} \sum \operatorname{dim} M_{m} q^{m}=\operatorname{tr}_{M} q^{-c / 24+L(0)} .
$$

*Supported by the Grants-in-Aids for Scientific Research, No. 09440004 and No. 10974001, The Ministry of Education, Science and Culture, Japan. 
In this paper, we consider these functions less formally by taking $q$ to be the usual local parameter $q=q_{\tau}=e^{2 \pi \imath \tau}$ at infinity in the upper half-plane

$$
\mathcal{H}=\{\tau \in \mathbb{C} \mid \Im \tau>0\} .
$$

Although it is often said that a vertex operator algebra (VOA) is a conformal field theory with mathematically rigorous axioms, the axioms of VOA do not assume the modular invariance. However, Zhu [Z] showed the modular $\left(S L_{2}(\mathbb{Z})\right)$ invariance of the space

$$
<q_{1}^{\left|a_{1}\right|} \cdots q_{n}^{\left|a_{n}\right|} \operatorname{tr}_{W} Y\left(a_{1}, q_{1}\right) \cdots Y\left(a_{n}, q_{n}\right) q^{L(0)-c / 24}: W \text { irr. } V-\bmod >
$$

for a rational VOA $V$ with central charge $c$ and $a_{i} \in V_{\left|a_{i}\right|}$ under Condition $C_{2}$, which are satisfied by many known examples, where $q_{j}=q_{z_{j}}=e^{2 \pi \imath z_{j}}$ and $\left|a_{i}\right|$ denotes the weight of $a_{j}$. For example, the space

$$
<\operatorname{ch}_{q} W: W \text { irreducible } V \text {-modules }>
$$

is $S L_{2}(\mathbb{Z})$-invariant. Recently, Dong, Li and Mason extended the Zhu's idea and proved a modular invariance of the space

$$
<\operatorname{tr}_{U} \phi^{i} q^{L(0)-c / 24}: i \in \mathbb{Z}, U \phi \text {-twisted modules }>
$$

by introducing the concept of $\phi$-twisted modules for a finite automorphism $\phi$, see [DLiM]. An easiest example of automorphism of VOA is given by a vector $v \in V_{1}$ as $\phi=e^{2 \pi v v(0)}$. Especially, if the eigenvalues of $o(v)(=v(0))$ on modules are in $\frac{1}{n} \mathbb{Z}$, then the order of $e^{2 \pi \imath o(v)}$ is finite. So for a $V$-module $W$ and $u, v \in V_{1}$, we will define

$$
Z_{W}(v ; u ; \tau)=\operatorname{tr}_{W} e^{2 \pi \imath\left(o(v)-\frac{\langle v, u\rangle}{2}\right)} q^{L(0)+o(u)-(c+12\langle u, u\rangle) / 24}
$$

and we call $Z_{W}(v ; 0 ; \tau) \eta(\tau)^{c}$ a theta-function of $W$, where $u_{1} u=-\langle u, u\rangle \mathbf{1}$ and $\eta(\tau)=q^{1 / 24} \prod_{n=1}^{\infty}\left(1-q^{n}\right)$ is the Dedekind $\eta$-function. It is worth noting that $c+12\langle u, u\rangle$ is the central charge of conformal element $\omega+L(-1) u$ and $o(\omega+L(-1) u)$ is equal to $L(0)+o(u)$, see DLnM.

For example, let $V$ is a lattice VOA $V_{2 \mathbb{Z} x}$ constructed from a 1-dimensional lattice $L=2 \mathbb{Z} x$ with $\langle x, x\rangle=1$. It has exactly four irreducible modules $[\mathbb{D}]$ :

$$
W_{0}=V_{2 \mathbb{Z} x}, W_{1}=V_{\left(2 \mathbb{Z}+\frac{1}{2}\right) x}, W_{2}=V_{(2 \mathbb{Z}+1) x}, W_{3}=V_{\left(2 \mathbb{Z}-\frac{1}{2}\right) x} .
$$

Let $\theta_{h, k}(z, \tau)\left(:=\sum_{n \in \mathbb{Z}} \exp \left(\pi \imath(n+k)^{2} \tau+2 \pi(n+k)(z+k)\right)\right)$ be theta functions for $h, k=0, \frac{1}{2}$. By the construction of lattice VOA (see [FLM]), it is easy to check

$$
\theta_{h, k}(z, \tau)=\eta(\tau)\left((\imath)^{4 h k} Z_{W_{2 h}}(z x(-1) \mathbf{1} ; 0 ; \tau)+(-1)^{k}(\imath)^{4 h k} Z_{W_{2+2 h}}(z x(-1) \mathbf{1} ; 0 ; \tau)\right)
$$

for $h, k=0, \frac{1}{2}$ and their modular transformations

$$
\theta_{h, k}(z / \tau,-1 / \tau)=(i)^{4 h k}(-\imath \tau)^{\frac{1}{2}} \exp \left(\pi \imath z^{2} / \tau\right) \theta_{k, h}(z, \tau)
$$

are well known, (see $\mathbb{M})$. In particular, there are constants $A_{k}^{h} \in \mathbb{C}$ such that

$$
Z_{W_{h}}(z x(-1) \mathbf{1} ; 0 ;-1 / \tau)=\sum A_{k}^{h} Z_{W_{h}}\left(0 ; z x(-1)+\frac{1}{2} z^{2}, \tau\right)
$$


Namely, the modular transformations of $Z_{W_{h}}(z x(-1) \mathbf{1} ; 0: \tau)$ are expressed by linear combinations of $Z_{W_{k}}(u ; v ; \tau)$ of (ordinary) modules $W_{k}$, but not twisted modules. By this result, for an automorphism $\phi=e^{v(0)}$, we can expect to obtain a modular transformation by using only the ordinary modules, which offers some information about twisted modules. This is the motivation of this paper and we will actually show that the above result is generally true, that is, we will prove that the following modular transformation by using Zhu's result (2).

Main Theorem Let $V$ be a rational vertex operator algebra with the irreducible modules $\left\{W_{i}: i=1, \ldots, m\right\}$ and $u, v \in V_{1}$. Assume $v(0) v=v(0) u=u(0) v=u(0) u=0$ and $v(1) v, v(1) u, u(1) u \in \mathbb{C} 1$. If $V$ satisfies Zhu's finite condition $C_{2}$, then

$$
\left\{Z_{W_{h}}(v ; u ; \tau): h=1, \ldots, m\right\}
$$

satisfy a modular invariance, i.e., for $\alpha=\left(\begin{array}{ll}a & b \\ f & d\end{array}\right) \in S L_{2}(\mathbb{Z})$, there are constants $A_{\alpha, k}^{h}$ (see Theorem 4.1) such that

$$
Z_{W_{h}}\left(v ; u ; \frac{a \tau+b}{f \tau+d}\right)=\sum_{k=1}^{m} A_{\alpha, k}^{h} Z_{W_{k}}(a v+b u ; f v+d u ; \tau) .
$$

\section{VOA}

A vertex operator algebra is a $\mathbb{Z}$-graded vector space:

$$
V=\oplus_{n \in \mathbb{Z}} V_{n}
$$

satisfying $\operatorname{dim} V_{n}<\infty$ for all $n$ and $V_{n}=0$ for $n<<0$, equipped with a linear map

$$
\begin{aligned}
& V \rightarrow(\operatorname{End} V)\left[\left[z, z^{-1}\right]\right] \\
& v \rightarrow Y(v, z)=\sum_{n \in \mathbb{Z}} v(n) z^{-n-1}
\end{aligned}
$$

and with two distinguished vectors, vacuum element $\mathbf{1} \in V_{0}$ and conformal vector $\omega \in V_{2}$ satisfying the following conditions for $u, v \in V$ :

$$
\begin{aligned}
& u(n) v=0 \quad \text { for } n \text { sufficiently large; } \\
& Y(\mathbf{1}, z)=1 ; \\
& Y(v, z) \mathbf{1} \in V[[z]] \text { and } \lim _{z \rightarrow 0} Y(v, z) \mathbf{1}=v ; \\
& (z-x)^{N} Y(v, z) Y(u, x)=(z-x)^{N} Y(u, x) Y(v, z) \quad \text { for } N \text { sufficiently large, }
\end{aligned}
$$

where $\left(z_{1}-z_{2}\right)^{n}(n \in \mathbb{Z})$ are to be expanded in nonnegative integral powers of second variable $z_{2}$

$$
[L(m), L(n)]=(m-n) L(m+n)+\frac{1}{12}(m 3-m) \delta_{m+n, 0} c
$$


for $m, n \in \mathbb{Z}$, where $L(m)=\omega(m+1)$ and $c$ is called central charge;

$$
\begin{aligned}
& L(0) v=n v \text { for } v \in V_{n} ; \\
& \frac{d}{d z} Y(v, z)=Y(L(-1) v, z) .
\end{aligned}
$$

This completes the definition.

We also have the notion of modules:

Let $(V, Y, \mathbf{1}, \omega)$ be a vertex operator algebra. A weak module $W$ of $(V, Y, \mathbf{1}, \omega)$ is a $\mathbb{C}$-graded vector space:

$$
W=\oplus_{n \in \mathbb{C}} W_{n}
$$

equipped with a linear map

$$
\begin{aligned}
V & \rightarrow(\operatorname{End}(W))\left[\left[z, z^{-1}\right]\right] \\
v & \rightarrow Y^{W}(v, z)=\sum_{n \in \mathbb{Z}} v^{W}(n) z^{-n-1} \quad\left(v_{n} \in \operatorname{End}(W)\right)
\end{aligned}
$$

satisfying the following conditions for $u, v \in V$ and $w \in W$ :

For $v \in V, w \in M, v^{W}(m) w=0$ for $m>>0$.

$$
\begin{gathered}
Y^{W}(\mathbf{1}, z)=1 ; \\
L^{W}(0) w=n w \text { for } w \in W_{n}, L^{W}(0)=\omega^{W}(1) ; \\
\frac{d}{d z} Y^{W}(v, z)=Y(L(-1) v, z)
\end{gathered}
$$

and the following Jacobi idenity holds.

$$
\begin{aligned}
& z_{0}^{-1} \delta\left(\frac{z_{1}-z_{2}}{z_{0}}\right) Y^{W}\left(u, z_{1}\right) Y^{W}\left(v, z_{2}\right)-z_{0}^{-1} \delta\left(\frac{z_{2}-z_{1}}{-z_{0}}\right) Y^{W}\left(v, z_{2}\right) Y^{W}\left(u, z_{1}\right) \\
& =z_{2}^{-1} \delta\left(\frac{z_{1}-z_{0}}{z_{2}}\right) Y^{W}\left(Y\left(u, z_{0}\right) v, z_{2}\right) .
\end{aligned}
$$

A weak module $W$ is called a module if every finitely generated weak submodule $M=$ $\oplus_{r \in \mathbb{C}} M_{r}$ of $W$ satisfies

(1) $\operatorname{dim} M_{r}<\infty$,

(2) $M_{r+n}=0$ for $n \in \mathbb{Z}$ sufficiently large, for any $r \in \mathbb{C}$.

\section{Formal power series}

We use the notation $q$ and $q_{z}$ to denote $e^{2 \pi \imath \tau}$ and $e^{2 \pi \imath z}$, respectively.

In this paper, the formal power series

$$
P_{2}\left(q_{z}, q\right)=(2 \pi \imath)^{2} \sum_{n=1}^{\infty}\left(\frac{n q_{z}^{n}}{1-q^{n}}+\frac{n q_{z}^{-n} q^{n}}{1-q^{n}}\right)
$$


plays an essential role, where $\frac{1}{1-q^{n}}$ is understood as $\sum_{i=0}^{\infty} q^{i}$. The limit of $P_{2}\left(q_{z}, q\right)$ (which we still denote as $\left.P_{2}\left(q_{z}, q\right)\right)$ relates to $\mathfrak{p}(z, \tau)$ by

$$
P_{2}\left(q_{z}, q\right)=\mathfrak{p}(z, \tau)+G_{2}(\tau)
$$

where

$$
G_{2}(\tau)=\frac{\pi^{2}}{2}+\sum_{m \in \mathbb{Z}-\{0\}} \sum_{n \in \mathbb{Z}} \frac{1}{(m \tau+n)^{2}}
$$

is the Eisenstein series and $\mathfrak{p}(z, \tau)$ is the Weierstrass $\mathfrak{p}$-function

$$
\mathfrak{p}(z, \tau)=\frac{1}{z^{2}}+\sum_{(m, n) \neq(0,0)}\left(\frac{1}{(z-m \tau-n)^{2}}-\frac{1}{(m \tau+n)^{2}}\right) .
$$

It is known that

$$
G_{2}\left(\frac{a \tau+b}{f \tau+d}\right)=(f \tau+d)^{2} G_{2}(\tau)-2 \pi \imath f(f \tau+d)
$$

and

$$
\mathfrak{p}\left(\frac{z}{f \tau+d}, \frac{a \tau+b}{f \tau+d}\right)=(f \tau+d)^{2} \mathfrak{p}(z, \tau)
$$

In particular,

$$
P_{2}\left(\frac{z}{f \tau+d}, \frac{a \tau+b}{f \tau+d}\right)=(f \tau+d)^{2} P_{2}(z, \tau)-2 \pi \imath f(f \tau+d) .
$$

In this paper, we will use variables $\left\{z_{1}, \ldots, z_{n}\right\}$ and calculate the products of formal power series $P_{2}\left(q_{z_{i}-z_{j}}, \tau\right)$. In order to simplify notation, we will use a transposition $(i, j)$ of symmetric groups $\Sigma_{n}$ on $\Omega=\{1, \ldots, n\}$. For $\left\{\left(i_{11}, i_{12}\right), \ldots,\left(i_{t 1}, i_{t 2}\right)\right\}$ with $i_{s 1}<i_{s 2}$ and $i_{a b} \neq i_{c d}$ for $(a, b) \neq$ $(c, d)$, we view $\sigma=\left(i_{11}, i_{12}\right) \cdots\left(i_{t 1}, i_{t 2}\right)$ as an involution (element of order 2$)$ of $\Sigma_{n}$ and denote $\prod_{j=1}^{t} P_{2}\left(q_{z_{t 2}-z_{t 1}}, \tau\right)$ by $\prod_{i<\sigma(i)} P_{2}\left(q_{z_{\sigma(i)}-z_{i}}, \tau\right)$. Let $I(n)$ denote the set of all elements $g$ in $\Sigma_{n}$ with $g^{2}=1$. For $\sigma \in \Sigma_{n}$, set

$$
\begin{aligned}
& m(\sigma)=\{i \in \Omega \mid \sigma(i) \neq i\} \\
& f(\sigma)=\{i \in \Omega \mid \sigma(i)=i\} .
\end{aligned}
$$

For $\sigma_{1}, \sigma_{2} \in \Sigma_{n}, \sigma_{1}, \sigma_{2}$ are called to be disjoint if $m\left(\sigma_{1}\right) \cap m\left(\sigma_{2}\right)=\emptyset$. $\sigma_{1}+\cdots+\sigma_{n}=\sigma$ expresses that $\left\{\sigma_{1}, \ldots, \sigma_{n}\right\}$ are mutually disjoint and the product $\sigma_{1} \cdots \sigma_{n}$ is equal to $\sigma$.

For $v \in V_{1}, v(0)$ acts on the finite dimensional homogeneous subspaces $V_{m}$ and satisfies $[v(0), v(m)]=(v(0) v)(m)$ and

$$
\begin{aligned}
v(0) \omega & =v(0) \omega(-1) \mathbf{1}=-[\omega(-1), v(0)] \mathbf{1}=-\sum_{i=0}^{\infty}(-1)^{i}(\omega(i) v)(-1-\imath) \mathbf{1} \\
& =-(\omega(0) v)(-1) \mathbf{1}+(\omega(1) v)(-2) \mathbf{1}=-v(-2) \mathbf{1}+(w(1) v)(-2) \mathbf{1}=0 .
\end{aligned}
$$

Therefore, $e^{v(0)}=\sum_{n=0}^{\infty} \frac{v(0)^{n}}{n !}$ is well defined and satifies that $e^{v(0)} \omega=\omega$ and $e^{v(0)}\left(u_{m} w\right)=$ $\left(e^{v(0)} u\right)_{m}\left(e^{v(0)} w\right)$. In particular, $e^{v(0)}$ is an automorphism of $V$. 
Definition 1 For $a V$-module $W$ and $u, v \in V_{1}$, define

$$
Z_{W}(v ; u ; \tau)=\operatorname{tr}_{W} e^{2 \pi \imath(v(0)-\langle v, u\rangle / 2)} q^{(u(0)-\langle u, u\rangle / 2)+L(0)-c / 24} .
$$

We set $\theta_{W}(v, \tau)=Z_{W}(v ; 0 ; \tau) \eta(\tau)^{c}$ and call it a theta-function of $W$, where $\eta(\tau)=q^{1 / 24} \prod_{n=1}^{\infty}\left(1-q^{n}\right)$ is the Dedekind $\eta$-function.

For example, let $V$ be a lattice VOA $V_{2 \mathbb{Z} x}$ associated with a 1-dimensional lattice $L=2 \mathbb{Z} x$ with $\langle x, x\rangle=1$, then

$$
W_{0}=V_{2 \mathbb{Z} x}, W_{1}=V_{\left(2 \mathbb{Z}+\frac{1}{2}\right) x}, W_{2}=V_{(2 \mathbb{Z}+1) x}, W_{3}=V_{\left(\mathbb{Z}-\frac{1}{2}\right) x}
$$

are the irreducible $V_{2 \mathbb{Z} x}$-modules by $[\mathrm{D}]$ and $\langle x(-1) \mathbf{1}, x(-1) \mathbf{1}\rangle=-1$. We then have

$$
\begin{aligned}
\theta_{0,0}(z, \tau) & =\sum_{n \in \mathbb{Z}} \exp \left(\pi \imath n^{2} \tau+2 \pi \imath n z\right) \\
& \left.=\theta_{W_{0}}(z x(-1) \mathbf{1}, \tau)+\theta_{W_{2}}(z x(-1) \mathbf{1}, \tau)\right) \\
\theta_{0, \frac{1}{2}}(z, \tau) & =\sum \exp \left(\pi \imath n^{2} \tau+2 \pi \imath n\left(z+\frac{1}{2}\right)\right) \\
& \left.=\theta_{W_{0}}(z x(-1) \mathbf{1}, \tau)-\theta_{W_{2}}(z x(-1) \mathbf{1}, \tau)\right) \\
\theta_{\frac{1}{2}, 0}(z, \tau) & =\sum \exp \left(\pi \imath\left(n+\frac{1}{2}\right)^{2} \tau+2 \pi \imath\left(n+\frac{1}{2}\right) z\right) \\
& \left.=\theta_{W_{1}}(z x(-1) \mathbf{1}, \tau)+\theta_{W_{3}}(z x(-1) \mathbf{1}, \tau)\right) \\
\theta_{\frac{1}{2}, \frac{1}{2}}(z, \tau) & =\sum \exp \left(\pi \imath\left(n+\frac{1}{2}\right)^{2} \tau+2 \pi \imath\left(n+\frac{1}{2}\right)\left(z+\frac{1}{2}\right)\right) \\
& \left.=\imath \theta_{W_{1}}(z x(-1) \mathbf{1}, \tau)-\imath \theta_{W_{3}}(z x(-1) \mathbf{1}, \tau)\right)
\end{aligned}
$$

and their modular transformations

$$
\begin{aligned}
& \theta_{0,0}(z / \tau,-1 / \tau)=(-\imath \tau)^{\frac{1}{2}} \exp \left(\pi \imath z^{2} / \tau\right) \theta_{0,0}(z, \tau) \\
& \theta_{0, \frac{1}{2}}(z / \tau,-1 / \tau)=(-\imath \tau)^{\frac{1}{2}} \exp \left(\pi \imath z^{2} / \tau\right) \theta_{1,0}(z, \tau) \\
& \theta_{\frac{1}{2}, 0}(z / \tau,-1 / \tau)=(-\imath \tau)^{\frac{1}{2}} \exp \left(\pi \imath z^{2} / \tau\right) \theta_{0,1}(z, \tau) \\
& \theta_{\frac{1}{2}, \frac{1}{2}}(z / \tau,-1 / \tau)=-(-\imath \tau)^{\frac{1}{2}} \exp \left(\pi \imath z^{2} / \tau\right) \theta_{1,1}(z, \tau)
\end{aligned}
$$

are well known, (see $\mathbb{M}$ ). Therefore, the modular transformations $Z_{W_{h}}\left(z x(-1) \mathbf{1} ; 0: \frac{-1}{\tau}\right)$ are expressed by linear combinations of $Z_{W_{k}}(u ; v ; \tau)$ of (ordinary) modules $W_{k}$, but not twisted modules.

\section{Modular invariance}

In this section, we will prove a modular invariance. Throughout this section, we assume : (A1) $V=\oplus_{n=0}^{\infty} V_{n}$ is a rational VOA; (A2) $\left\{W^{1}, \ldots, W^{m}\right\}$ is the set of all irreducible $V$-modules; 
(A3) fix $v_{1}, \ldots, v_{n} \in V_{1}$ satisfying $v_{r}(0) v_{j}=0$ and $v_{r}(1) v_{j} \in \mathbb{C} 1$ for any $r, j$.

By (A3), we have

$$
\left[v_{r}(m), v_{j}(n)\right]=\sum\left(\begin{array}{c}
m \\
k
\end{array}\right)\left(v_{r}(k) v_{j}\right)(m+n-\imath)=m\left(v_{r}(1) v_{j}\right)(m+n-1)=\delta_{m,-n} m\left\langle-v_{r}, v_{j}\right\rangle,
$$

where $v_{r}(1) v_{j}=\left\langle-v_{r}, v_{i}\right\rangle \mathbf{1}$.

For a grade-keeping endomorphism formal power series $\psi \in \operatorname{End}(W)\left(\left(q_{z_{1}}, \ldots, q_{z_{n}}, q_{\tau}\right)\right)$ of $W$, set

$$
S_{W}\left(\psi ; z_{1}, \ldots, z_{n}, \tau\right)=\operatorname{tr}_{W} \psi Y\left(v_{1}, q_{z_{1}}\right) \cdots Y\left(v_{n}, q_{z_{n}}\right) q_{z_{1}} \cdots q_{z_{n}} q^{L(0)-\frac{c}{24}} .
$$

By the same argument as in the proof of Proposition 4.3.2 in [Z], we have the following:

Proposition 4.1 Assume $\left[\psi, v_{r}(n)\right]=0$ for $r=1, \ldots, n$. Then we have:

$$
S_{W}\left(\psi ; z_{1}, z_{2}, \ldots, z_{n}, \tau\right)=\sum_{\sigma \in I(n)} S_{W}\left(\psi \prod_{r \in f(\sigma)} o\left(v_{r}\right) \prod_{j<\sigma(j)}\left(\left\langle-v_{j}, v_{\sigma(j)}\right\rangle \frac{P_{2}\left(z_{\sigma(j)} z_{j}^{-1}, \tau\right)}{(2 \pi \imath)^{2}}\right) ; \tau\right)
$$

where $I(n)$ is the set of all elements $\sigma$ in the symmetric group $\Sigma_{n}$ on $n$ point set $\Omega=\{1,2, \ldots, n\}$ with $\sigma^{2}=1$ and $f(\sigma)$ denotes the set of fixed points of $\sigma$.

[Proof $\quad$ For $k \in \mathbb{Z}$, we have

$$
\begin{aligned}
& S_{W}\left(\psi v_{1}(k) q_{z_{1}}^{-k} ; z_{2}, \ldots, z_{n}, \tau\right) \\
= & \operatorname{tr}_{W} \psi v_{1}(k) q_{z_{1}}^{-k} Y\left(v_{2}, q_{z_{2}}\right) \cdots Y\left(v_{n}, q_{z_{n}}\right) q_{z_{1}} \cdots q_{z_{n}} q^{L(0)-c / 24} \\
= & \operatorname{tr}_{W} \psi\left[v_{1}(k), Y\left(v_{2}, q_{z_{2}}\right) \cdots Y\left(v_{n}, q_{z_{n}}\right)\right] q_{z_{1}}^{-k} q_{z_{2}} \cdots q_{z_{n}} q^{L(0)-c / 24} \\
& +\operatorname{tr}_{W} \psi Y\left(v_{2}, q_{z_{2}}\right) \cdots Y\left(v_{n}, q_{z_{n}}\right) v(k) q_{z_{1}}^{-k} q_{z_{2}} \cdots q_{z_{n}} q^{L(0)-c / 24} \\
= & \sum_{j=2}^{n} \sum_{i \in \mathbb{N}}\left(\begin{array}{c}
k \\
i
\end{array}\right) q_{z_{j}}^{k-\imath} \operatorname{tr}_{W} \psi Y\left(v_{2}, q_{z_{2}}\right) \cdots Y\left(v_{1}(i) v_{j}, q_{z_{j}}\right) \cdots Y\left(v_{n}, q_{z_{n}}\right) q_{z_{1}}^{-k} q_{z_{2}} \cdots q_{z_{n}} q^{L(0)-c / 24} \\
& +\operatorname{tr}_{W} \psi Y\left(v_{2}, q_{z_{2}}\right) \cdots Y\left(v_{n}, q_{z_{n}}\right) q_{z_{1}}^{-k} q_{z_{2}} \cdots q_{z_{n}} q^{L(0)-c / 24} v(k) q^{k} \\
= & \sum_{j=2}^{n} k q_{z_{j}}^{k-1} q_{z_{1}}^{-k_{\operatorname{tr}}} \operatorname{tr}_{W} \psi\left(v_{2}, q_{z_{2}}\right) \cdots Y\left(\left\langle-v_{j}, v_{1}\right\rangle \mathbf{1}, q_{z_{j}}\right) \cdots Y\left(v_{n}, q_{z_{n}}\right) q_{z_{2}} \cdots q_{z_{n}} q^{L(0)-c / 24} \\
& +\operatorname{tr}_{W} \psi v_{1}(k) q_{z_{1}}^{-k} Y\left(v_{2}, q_{z_{2}}\right) \cdots Y\left(v_{n}, q_{z_{n}}\right) q_{z_{2}} \cdots q_{z_{n}} q^{L(0)-c / 24} q^{k} \\
= & \left.\sum_{j=2}^{n} \operatorname{tr}_{W}\left\langle-v_{j}, v_{1}\right\rangle k q_{z_{j}-z_{1}}^{k} \psi Y\left(v_{2}, q_{z_{2}}\right) \cdots Y \widehat{\left(v_{j}, q_{z_{j}}\right.}\right) \cdots Y\left(v_{n}, q_{z_{n}}\right) q_{z_{2}} \cdots \widehat{q_{z_{j}} \cdots q_{z_{n}} q^{L(0)-c / 24}} \\
& +\operatorname{tr}_{W} \psi v_{1}(k) q_{z_{1}}^{-k} Y\left(v_{2}, q_{z_{2}}\right) \cdots Y\left(v_{n}, q_{z_{n}}\right) q_{z_{2}} \cdots q_{z_{n}} q^{L(0)-c / 24} q^{k} \\
= & \left.\sum_{j=2}^{n} \operatorname{tr}_{W}\left\langle-v_{j}, v_{1}\right\rangle k q_{z_{j}-z_{1}}^{k} \psi Y\left(v_{2}, q_{z_{2}}\right) \cdots Y \widehat{\left(v_{j}, q_{z_{j}}\right.}\right) \cdots Y\left(v_{n}, q_{z_{n}}\right) q_{z_{2}} \cdots \widehat{q_{z_{j}}} \cdots q_{z_{n}} q^{L(0)-c / 24} \\
& +\operatorname{tr}_{W} \psi v_{1}(k) q_{z_{1}}^{-k} Y\left(v, q_{z_{2}}\right) \cdots Y\left(v, q_{z_{n}}\right) q_{z_{2}} \cdots q_{z_{n}} q^{L(0)-c / 24} q^{k} \\
= & \sum_{j=2}^{n}\left\langle-v_{1}, v_{j}\right\rangle k q_{z_{j}-z_{1}}^{k} S\left(\psi ; z_{2}, \ldots, \widehat{z_{j}}, \ldots, z_{n}, \tau\right)+S\left(\psi v_{1}(k) q_{z_{1}}^{-k} ; z_{2}, \ldots, z_{n}, \tau\right) q^{k}, \\
&
\end{aligned}
$$


where $\widehat{q_{z_{j}}}$ means that we take off the term $q_{z_{j}}$.

Hence, for $k \neq 0$, we have:

$$
S_{W}\left(\psi v_{1}(k) q_{z_{1}}^{-k} ; z_{2}, \ldots, z_{n}, \tau\right)=\sum_{j=2}^{n}\left\langle-v_{j}, v_{1}\right\rangle \frac{k q_{z_{j}-z_{1}}^{k}}{1-q^{k}} S_{W}\left(\psi ; z_{2}, \ldots, z_{j-1}, z_{j+1}, \ldots, z_{n}, \tau\right) .
$$

Therefore, we have:

$$
\begin{aligned}
& S_{W}\left(\psi ; z_{1}, z_{2}, \ldots, z_{n}, \tau\right) \\
= & S_{W}\left(\psi v_{1}(0) ; z_{2}, \ldots, z_{n}, \tau\right)+\sum_{k \neq 0} S_{W}\left(\psi v(k) q_{z_{1}}^{-k} ; z_{2}, \ldots, z_{n}, \tau\right) \\
= & S_{W}\left(\psi v_{1}(0) ; z_{2}, \ldots, z_{n}, \tau\right)+\sum_{k \neq 0} \sum_{j=2}^{n}\left\langle-v_{j}, v_{1}\right\rangle \frac{k q_{z_{j}-z_{1}}^{k}}{1-q^{k}} S_{W}\left(\psi ; z_{2}, \ldots, z_{j-1}, z_{j+1}, \ldots, z_{n}, \tau\right) \\
= & S_{W}\left(\psi v_{1}(0) ; z_{2}, \ldots, z_{n}, \tau\right)-\sum_{j=2}^{n}\left\langle v_{u}, v_{1}\right\rangle \frac{P_{2}\left(q_{z_{j}-z_{1}}, \tau\right)}{(2 \pi \imath)^{2}} S_{W}\left(\psi ; z_{2}, \ldots, z_{j-1}, z_{j+1}, \ldots, z_{n}, \tau\right)
\end{aligned}
$$

By substituting $\psi v_{1}(0)$ into $\phi$ and repeating these steps, we have:

$$
S_{W}\left(\psi ; z_{1}, z_{2}, \ldots, z_{n}, \tau\right)=\sum_{\sigma \in I(n)} \prod_{i<\sigma(i)}\left(\left\langle-v_{i}, v_{\sigma(i)}\right\rangle \frac{P_{2}\left(q_{z_{\sigma(i)}-z_{i}}, \tau\right)}{(2 \pi \imath)^{2}}\right) S_{W}\left(\psi \prod_{i \in f(\sigma)} v_{i}(0), \tau\right) .
$$

Q.E.D.

The main result we quote from [B] is

Theorem 4.1 (Zhu) Let $(V, Y, \mathbf{1}, \omega)$ be a rational VOA satisfying (A1) and (A2). Then for any $\alpha=\left(\begin{array}{ll}a & b \\ f & d\end{array}\right) \in S L_{2}(\mathbb{Z})$, we have

$$
S_{W_{h}}\left(1, \frac{z_{1}}{f \tau+d}, \ldots, \frac{z_{n}}{f \tau+d}, \frac{a \tau+b}{f \tau+d}\right)=(f \tau+d)^{n} \sum_{j=1}^{m} A_{\alpha, k}^{h} S_{W_{k}}\left(1 ; z_{1}, \ldots, z_{n}, \tau\right)
$$

where the $A_{\alpha, k}^{h}$ are constants depending only on $\alpha, h, k$.

To simplify notation, $S_{k}(\cdots), \alpha(v)$ and $d(\alpha)$ denote $S_{W_{k}}(\cdots),(f \tau+d) o(v)$ and $f \tau+d$, respectively. Set

$$
\begin{aligned}
D(r, j) & =\left\langle-v_{r}, v_{j}\right\rangle\left(\frac{1}{2 \pi \imath}\right)^{2}(d(\alpha))^{2} P_{2}\left(q_{z_{r}-z_{j}}, \tau\right), \\
E(r, j) & =\left\langle-v_{r}, v_{j}\right\rangle\left((d(\alpha))^{2} P_{2}\left(q_{z_{r}-z_{j}}, \tau\right)-\left(\frac{1}{2 \pi \imath}\right) f(d(\alpha))\right), \\
D_{\sigma}= & \prod_{j<\sigma(j)} D(\sigma(j), j), \text { and } \\
E_{\sigma}= & \prod_{j<\sigma(j)} E(\sigma(j), j) .
\end{aligned}
$$


Lemma 4.1 If $|m(\sigma)|=2 p$, then

$$
\sum_{\sigma_{1}+\ldots+\sigma_{t}=\sigma}(-1)^{t} E_{\sigma_{t}} \cdots E_{\sigma_{2}} E_{\sigma_{1}}=(-1)^{p} E_{\sigma}
$$

[Proof] We first note that $E_{\sigma_{t}} \cdots E_{\sigma_{2}} E_{\sigma_{1}}=E_{\sigma}$. Therefore, we have to count the number of $E_{\sigma}$ in the left side. We will prove it by induction on $p$. If $p=1$, then it is trivial. For $\sigma=\left(r_{1}, r_{2}\right) \cdots\left(r_{2 p-1}, r_{2 p}\right)$, the number of $\sigma_{1}$ with $\sigma_{1}+\cdots+\sigma_{t}=\sigma$ and $\left|m\left(\sigma_{1}\right)\right|=2 r$ is $\left(\begin{array}{l}p \\ r\end{array}\right)$. Therefore, by induction, we have:

$$
\sum_{\sigma_{1}+\ldots+\sigma_{t}=\sigma}(-1)^{t} E_{\sigma_{t}} \cdots E_{\sigma_{2}} E_{\sigma_{1}}=-\sum_{j=1}^{p}\left(\begin{array}{l}
p \\
j
\end{array}\right)(-1)^{p-j} E_{\sigma}=-\left(-(-1)^{p}\right) E_{\sigma}=(-1)^{p} E_{\sigma} .
$$

Q.E.D.

For $\alpha=\left(\begin{array}{ll}a & b \\ f & d\end{array}\right) \in S L_{2}(\mathbb{Z})$, we have

\section{Lemma 4.2}

$$
S_{h}\left(\prod_{r=1}^{n} o\left(v_{r}\right) ; \frac{a \tau+b}{f \tau+d}\right)=\sum_{k=1}^{m} A_{\alpha, k}^{h} \sum_{\sigma \in I(n)} \prod_{j<\sigma(j)}\left(\frac{f(d(\alpha))}{2 \pi \imath}\right)\left\langle-v_{j}, v_{\sigma(j)}\right\rangle S_{k}\left(\prod_{r \in f(\sigma)} \alpha\left(v_{r}\right) ; \tau\right) .
$$

[Proof] By Theorem 3.1 and Proposition 3.1, we have:

$$
\begin{aligned}
& \sum_{\sigma \in I(n)}\left(\prod_{r<\sigma(r)}\left(\left\langle-v_{\sigma(r)}, v_{r}\right\rangle\left(\frac{1}{2 \pi \imath}\right)^{2} P_{2}\left(\frac{q_{\frac{z_{\sigma(r)}-z_{r}}{f \tau+d}}}{f}, \frac{a \tau+b}{f \tau+d}\right)\right)\right) S_{h}\left(\prod_{j \in f(\sigma)} o\left(v_{j}\right), \frac{a \tau+b}{f \tau+d}\right) \\
= & S_{h}\left(1 ; \frac{z_{1}}{f \tau+d}, \ldots, \frac{z_{n}}{f \tau+d}, \frac{a \tau+b}{f \tau+d}\right) \\
= & (d(\alpha))^{n} \sum_{k=1}^{m} A_{\alpha, k}^{h} S_{k}\left(1 ; z_{1}, \ldots, z_{n}, \tau\right) \\
= & (d(\alpha))^{n} \sum_{k=1}^{m} A_{\alpha, k}^{h} \sum_{\sigma \in I(n)}\left(\prod_{r<\sigma(r)}\left(\left\langle-v_{r}, v_{\sigma(r)}\right\rangle\left(\frac{1}{2 \pi \imath}\right)^{2} P_{2}\left(q_{z_{\sigma(r)}-z_{r}}, \tau\right)\right)\right) S_{k}\left(\prod_{j \in f(\sigma)} o\left(v_{j}\right) ; \tau\right) .
\end{aligned}
$$

Hence, we have

$$
\begin{aligned}
& S_{h}\left(\prod_{r=1}^{n} o\left(v_{r}\right) ; \frac{a \tau+b}{f \tau+d}\right) \\
= & \left.\sum_{k=1}^{m} A_{\alpha, k}^{h} \sum_{\sigma \in I(n)}\left(\prod_{r<\sigma(r)}\left(\left\langle-v_{r}, v_{\sigma(r)}\right\rangle(d(\alpha))^{2}\left(\frac{1}{2 \pi \imath}\right)^{2} P_{2}\left(q_{z_{\sigma(r)}-z_{r}}, \tau\right)\right)\right) S_{k}\left(\prod_{j \in f(\sigma)} \alpha\left(v_{j}\right)\right), \tau\right)
\end{aligned}
$$




$$
\begin{aligned}
& -\sum_{1 \neq \sigma \in I(n)}\left(\prod_{r<\sigma(r)}\left(\left\langle-v_{r}, v_{\sigma(r)}\right\rangle\left((d(\alpha))^{2}\left(\frac{1}{2 \pi \imath}\right)^{2} P_{2}\left(q_{z_{\sigma(r)}-z_{r}}, \tau\right)-\left(\frac{1}{2 \pi \imath}\right) f(d(\alpha))\right)\right) \times\right. \\
& \times S_{h}\left(\prod_{j \in f(\sigma)} o\left(v_{j}\right) ; \frac{a \tau+b}{f \tau+d}\right) \\
= & \sum_{k=1}^{m} A_{\alpha, k}^{h} \sum_{\sigma \in I(n)}\left(\prod_{r<\sigma(r)} D(\sigma(r), r)\right) S_{k}\left(\prod_{j \in f(\sigma)} \alpha\left(v_{j}\right), \tau\right) \\
& -\sum_{1 \neq \sigma \in I(n)}\left(\prod_{r<\sigma(r)} E(\sigma(r), r)\right) S_{h}\left(\prod_{j \in f(\sigma)} o\left(v_{j}\right) ; \frac{a \tau+b}{f \tau+d}\right) \\
= & \sum_{k=1}^{m} A_{\alpha, k}^{h} \sum_{\sigma \in I(n)} D_{\sigma} S_{k}\left(\prod_{j \in f(\sigma)} \alpha\left(v_{j}\right) ; \tau\right)-\sum_{1 \neq \sigma \in I(n)} E_{\sigma} S_{h}\left(\prod_{r \in f(\sigma)} o\left(v_{r}\right) ; \frac{a \tau+b}{f \tau+d}\right) .
\end{aligned}
$$

Substituting the above equation into the last term, we have

$$
\begin{aligned}
& S_{h}\left(\prod_{r=1}^{n} o\left(v_{r}\right) ; \frac{a \tau+b}{f \tau+d}\right) \\
= & \sum_{k=1}^{m} A_{\alpha, k}^{h} \sum_{\sigma \in I(n)} D_{\sigma} S_{k}\left(\prod_{j \in f(\sigma)} \alpha\left(v_{j}\right) ; \tau\right) \\
& -\sum_{1 \neq \sigma \in I(n)} E_{\sigma} \sum_{k=1}^{m} A_{\alpha, k}^{h} \sum_{\sigma^{\prime} \in I(n), m\left(\sigma^{\prime}\right) \cap m(\sigma)=\emptyset} D_{\sigma^{\prime}} S_{k}\left(\prod_{r \in f\left(\sigma^{\prime}+\sigma\right)} \alpha\left(v_{r}\right) ; \tau\right) \\
& \left.+\sum_{1 \neq \sigma \in I(n)} E_{\sigma} \sum_{1 \neq \sigma^{\prime} \in I(n), m\left(\sigma^{\prime}\right) \cap m(\sigma)=\emptyset} E_{\sigma^{\prime}} S_{h}\left(\prod_{j \in f(\sigma) \cap f\left(\sigma^{\prime}\right)} o\left(v_{j}\right) ; \frac{a \tau+b}{f \tau+d}\right)\right\} .
\end{aligned}
$$

Repeating the above steps and by the equations

$$
\begin{aligned}
& S_{h}\left(o(v) ; \frac{a \tau+b}{f \tau+d}\right)=\sum A_{\alpha, k}^{h} S_{k}(o(v) ; \tau) \\
& S_{h}\left(1 ; \frac{a \tau+b}{f \tau+d}\right)=\sum A_{\alpha, k}^{h} S_{k}(1 ; \tau) .
\end{aligned}
$$

in [4], we have

$$
\begin{aligned}
& S_{h}\left(\prod_{r=1}^{n} o\left(v_{r}\right) ; \frac{a \tau+b}{f \tau+d}\right) \\
& =\sum_{k=1}^{m} A_{\alpha, k}^{h} S_{k}\left(\sum_{\sigma \in I(n)} D_{\sigma} \prod_{j \in f(\sigma)} \alpha\left(v_{j}\right) ; \tau\right) \\
& \quad-\sum_{1 \neq \sigma \in I(n)} E_{\sigma} S_{h}\left(\prod_{r \in f(\sigma)} o\left(v_{r}\right) ; \frac{a \tau+b}{f \tau+d}\right)
\end{aligned}
$$




$$
\begin{aligned}
& =\sum_{k=1}^{m} A_{\alpha, k}^{h}\left\{\sum_{\sigma_{1}, \ldots, \sigma_{t} \in I(n):\left|f\left(\sigma_{2}+\ldots \sigma_{t}\right)\right| \leq n-2}(-1)^{t-1} E_{\sigma_{t}} \cdots E_{\sigma_{2}} D_{\sigma_{1}} S_{k}\left(\prod_{r \in \cap_{j=1}^{t} f\left(\sigma_{j}\right)} \alpha\left(v_{r}\right) ; \tau\right)\right. \\
& \left.(\text { if } n \text { is odd })-\sum_{j=1}^{n} \sum_{\sigma_{1}, \ldots, \sigma_{t} \in I(n): f\left(\sigma_{1}+\ldots+\sigma_{t}\right)=\{j\}}(-1)^{t} E_{\sigma_{t}} \cdots E_{\sigma_{2}} E_{\sigma_{1}} S_{k}\left(\alpha\left(v_{j}\right) ; \tau\right)\right\} \\
& \text { (if } \left.n \text { is even) }-\sum_{\sigma_{1}, \ldots, \sigma_{t} \in I(n): f\left(\sigma_{1}+\ldots+\sigma_{t}\right)=\emptyset}(-1)^{t} E_{\sigma_{t}} \cdots E_{\sigma_{2}} E_{\sigma_{1}} S_{k}(1 ; \tau)\right\}
\end{aligned}
$$

by Lemma 3.1

$$
\begin{aligned}
& =\sum_{k=1}^{m} A_{\alpha, k}^{h}\left\{\sum_{\sigma \in I(n)} \sum_{\sigma_{1}, \sigma_{2} \in I(n): \sigma_{1}+\sigma_{2}=\sigma,\left|f\left(\sigma_{1}\right)\right| \geq 2}(-1)^{\left|\sigma_{1}\right| / 2} E_{\sigma_{1}} D_{\sigma_{2}} S_{k}\left(\prod_{r \in f(\sigma)} \alpha\left(v_{r}\right) ; \tau\right)\right. \\
& \text { (if } \left.n \text { is odd) }-\sum_{j=1}^{n} \sum_{\sigma \in I(n): f(\sigma)=\{j\}}(-1)^{(n-1) / 2} E_{\sigma} S_{k}\left(\alpha\left(v_{j}\right) ; \tau\right)\right\} \\
& \text { (if } \left.n \text { is even) }-\sum_{\sigma \in I(n): f(\sigma)=\emptyset}(-1)^{n / 2} E_{\sigma} S_{k}(1 ; \tau)\right\} \\
& =\sum_{k=1}^{m} A_{\alpha, k}^{h}\left\{\sum_{\sigma \in I(n)} \prod_{j<\sigma(j)}\left(\frac{1}{2 \pi \imath}\right)\left(f(d(\alpha))\left\langle-v_{j}, v_{\sigma(j)}\right\rangle\right)^{p} S_{k}\left(\prod_{r \in f(\sigma)} \alpha\left(v_{r}\right) ; \tau\right)\right\}
\end{aligned}
$$

Q.E.D.

Theorem A For $\alpha=\left(\begin{array}{ll}a & b \\ f & d\end{array}\right) \in S L_{2}(\mathbb{Z})$, we have

$$
S_{h}\left(e^{o(v)} ; \frac{a \tau+b}{f \tau+d}\right)=\sum_{k=1}^{m} A_{\alpha, k}^{h} S_{k}\left(e^{\frac{\langle-v, v\rangle}{2}\left(\frac{1}{2 \pi \imath}\right) f(d(\alpha))+(d(\alpha)) o(v)}, \tau\right) .
$$

In particular,

$$
Z_{W_{h}}\left(v ; 0 ; \frac{a \tau+b}{f \tau+d}\right)=\sum_{k=1}^{m} A_{\alpha, k}^{h} Z_{W_{k}}(d v ; f v ; \tau)
$$

[Proof] Let's calculate the coefficient of $\left(\langle-u, v\rangle\left(\frac{1}{2 \pi \imath}\right) f(d(\alpha))\right)^{p}(\alpha(v))^{r}$ in $(4.2)$ by setting $n=2 p+r$ and $v_{1}=\cdots=v_{n}=v$.

The number of involutions $\sigma$ with $|f(\sigma)|=r$ is $\left(\begin{array}{c}2 p+r \\ r\end{array}\right) \times \frac{(2 p+r) !}{p ! 2^{p}}$. Therefore, we have

$$
\begin{aligned}
& S_{h}\left(e^{o(v)} ; \frac{a \tau+b}{f \tau+d}\right)=S_{h}\left(\sum_{n=0}^{\infty} \frac{1}{n !} \prod_{i=1}^{n} o(v) ; \frac{a \tau+b}{f \tau+d}\right)=\sum_{n=0}^{\infty} \frac{1}{n !} S_{h}\left(\prod_{i=1}^{n} o(v) ; \frac{a \tau+b}{f \tau+d}\right) \\
& =\sum_{r, p \in \mathbb{N}} \frac{1}{n !} \sum_{k=1}^{m} A_{\alpha, k}^{h} \sum_{\sigma \in I(n)}\left(\langle-v, v\rangle \frac{f(d(\alpha))}{2 \pi \imath}\right)^{|m(\sigma)| / 2} S_{k}\left((\alpha(v))^{|f(\sigma)|} ; \tau\right) \\
& =\sum_{k=1}^{m} A_{\alpha, k}^{h} \sum_{r, p \in \mathbb{N}} \frac{1}{(r+2 p) !}\left(\begin{array}{c}
r+2 p \\
r
\end{array}\right) \frac{(2 p) !}{p ! 2^{p}}\left(\langle-v, v\rangle \frac{f(d(\alpha))}{2 \pi \imath}\right)^{|m(\sigma)| / 2} S_{k}\left((\alpha(v))^{|f(\sigma)|} ; \tau\right)
\end{aligned}
$$




$$
\begin{aligned}
& =\sum_{k=1}^{m} A_{\alpha, k}^{h} \sum_{r, p \in \mathbb{N}} \frac{1}{(r+p) !}\left(\begin{array}{c}
r+p \\
p
\end{array}\right)\left(\frac{\langle-v, v\rangle}{2} \frac{f(d(\alpha))}{2 \pi \imath}\right)^{p} S_{k}\left((\alpha(v))^{r} ; \tau\right) \\
& =\sum_{k=1}^{m} A_{\alpha, k}^{h} \sum_{n=0}^{\infty} \sum_{r=0}^{n} \frac{1}{n !}\left(\begin{array}{c}
n \\
p
\end{array}\right)\left(\frac{\langle-v, v\rangle}{2} \frac{f(d(\alpha))}{2 \pi \imath}\right)^{n-r} S_{k}\left((\alpha(v))^{r} ; \tau\right) \\
& =\sum_{r=1}^{m} A_{\alpha, k}^{h} S_{k}\left(\sum_{n=0}^{\infty} \frac{1}{n !}\left(\left(\frac{\langle-v, v\rangle}{2} \frac{f(d(\alpha))}{2 \pi \imath}\right)+\alpha(v)\right)^{n} ; \tau\right) \\
& =\sum_{r=1}^{m} A_{\alpha, k}^{h} S_{k}\left(e^{\frac{\langle-v, v\rangle}{2} \frac{f(d(\alpha))}{2 \pi \imath}+\alpha(v)} ; \tau\right),
\end{aligned}
$$

since

$$
\frac{1}{(r+2 p) !}\left(\begin{array}{c}
r+2 p \\
r
\end{array}\right) \frac{(2 p) !}{p ! 2^{p}}=\frac{1}{(r+2 p) !} \frac{(r+2 p) !}{k !(2 p) !} \frac{(2 p) !}{p ! 2^{p}}=\frac{1}{(r+p) !}\left(\begin{array}{c}
r+p \\
r
\end{array}\right) \frac{1}{2^{p}} .
$$

Q.E.D.

Let's show the final version of our result.

Main Theorem For $\alpha=\left(\begin{array}{ll}a & b \\ f & d\end{array}\right) \in S L_{2}(\mathbb{Z})$, we have

$$
Z_{W_{h}}\left(v ; u ; \frac{a \tau+b}{f \tau+d}\right)=\sum_{k=1}^{m} A_{\alpha, k}^{h} Z_{W_{k}}(d v+b u ; f v+a u ; \tau),
$$

where $A_{\alpha, k}^{h}$ are the coefficients in the equations given by Zhu [Z].

[Proof] Fix $s, t \in \mathbb{N}$ so that $n=s+t$. Assume that

$$
v_{1}=v_{2}=\ldots=v_{s},(\text { say, }=v), \quad u_{s+1}=\cdots=v_{s+t}, \quad(\text { say, }=u) .
$$

For $p, q, r \in \mathbb{Z}$, set $k=s-2 p-r \geq 0, h=t-2 q-r \geq 0$.

In order to simplify notation, for $u, v \in V_{1}$ and $\sigma \in I(n)$, we will use the following notation:

$$
\begin{aligned}
& \alpha(-v, u)=\frac{f(d(\alpha))}{2 \pi \imath}\langle-v, u\rangle, \\
& \alpha(v)=(d(\alpha)) o(v) \\
& m 11 \sigma=|\{r \in \Omega \mid r<\sigma(r) \leq s\}|, \\
& m 12 \sigma=|\{r \in \Omega \mid r \leq s<\sigma(r)\}|, \\
& m 22 \sigma=|\{r \in \Omega \mid s<r<\sigma(i)\}|, \\
& f 1 \sigma=|\{r \in \Omega \mid r=\sigma(r) \leq s\}| \text { and } \\
& f 2 \sigma=|\{r \in \Omega \mid s<i=\sigma(r)\}| .
\end{aligned}
$$


Then by lemma 4.2 , we have

$$
\begin{aligned}
& S_{h}\left(o(v)^{s} o(u)^{t} ; \frac{a \tau+b}{f \tau+d}\right) \\
= & \sum_{g=1}^{m} A_{\alpha, g}^{h} \sum_{\sigma \in I(s+t)} \alpha(-v, v)^{m 11 \sigma} \alpha(-v, u)^{m 12 \sigma} \alpha(-u, u)^{m 22 \sigma} S_{g}\left(\alpha(v)^{f 1 \sigma} \alpha(u)^{f 2 \sigma} ; \tau\right) .
\end{aligned}
$$

Hence,

$$
\begin{aligned}
& S_{h}\left(o(v)^{s} o(u)^{t} ; \frac{a \tau+b}{f \tau+d}\right) \\
= & \sum_{g=1}^{m} A_{\alpha, g}^{h} \sum_{r, p, q} \frac{(2 p+r+k) !(2 q+r+h) !}{k ! h ! r ! p ! q ! 2^{p+q}} \alpha(-v, v)^{p} \alpha(-v, u)^{r} \alpha(-u, u)^{q} S_{g}\left(\alpha(v)^{k} \alpha(u)^{h} ; \tau\right) .
\end{aligned}
$$

Expanding the exponential, we have:

$$
\begin{aligned}
& S_{h}\left(e^{2 \pi \imath(o(v)+\langle-v, u\rangle / 2)+2 \pi \imath \tau(o(u)+\langle-u, u\rangle / 2)} ; \tau\right) \\
& =\operatorname{tr}_{W_{h}} \sum_{n=0}^{\infty} \frac{(2 \pi \imath)^{n}}{n !}\left(o(v)+\tau o(u)+\frac{\langle-v, u\rangle}{2}+\tau \frac{\langle-u, u\rangle}{2}\right)^{n} q^{L(0)-c / 24} \\
& =\operatorname{tr}_{W_{h}} \sum_{\alpha, \beta, \gamma, \delta} \frac{(2 \pi \imath)^{\alpha+\beta+\gamma+\delta}}{\alpha ! \beta ! \gamma ! \delta !} o(v)^{\alpha}(\tau o(u))^{\beta}\left(\frac{\langle-v, u\rangle}{2}\right)^{\gamma}\left(\frac{\langle-u, u\rangle}{2} \tau\right)^{\delta} q^{L(0)-c / 24} .
\end{aligned}
$$

Hence, for $\alpha=\frac{-1}{\tau}$, we have:

$$
\begin{aligned}
& S_{h}\left(e^{2 \pi i(o(v)+\langle-v, u\rangle / 2+\tau(o(u)+\langle-u, u\rangle / 2))} ; \frac{-1}{\tau}\right) \\
= & \sum_{\alpha, \beta, \gamma, \delta} \frac{(2 \pi \imath)^{\alpha+\beta+\gamma+\delta}}{\alpha ! \beta ! \gamma ! \delta !} S_{h}\left(o(v)^{\alpha}\left(\frac{-1}{\tau} o(u)\right)^{\beta}\left(\frac{\langle-v, u\rangle}{2}\right)^{\gamma}\left(\frac{\langle-u, u\rangle}{2}\right)^{\delta}\left(\frac{-1}{\tau}\right)^{\delta}, \frac{-1}{\tau}\right) \\
= & \sum_{\gamma, \delta, \alpha, \beta} \frac{(2 \pi \imath)^{\alpha+\beta+\gamma+\delta}}{\alpha ! \beta ! \gamma ! \delta !}\left(\frac{\langle-u, u\rangle}{2}\right)^{\delta}\left(\frac{-1}{\tau}\right)^{\delta}\left(\frac{\langle-v, u\rangle}{2}\right)^{\gamma}\left(\frac{-1}{\tau}\right)^{\beta} S_{h}\left(o(v)^{\alpha} o(u)^{\beta} ; \frac{-1}{\tau}\right) \\
= & \sum_{\gamma, \delta, \alpha, \beta} \frac{(2 \pi \imath)^{\alpha+\beta+\gamma+\delta}}{\alpha ! \beta ! \gamma ! \delta !}\left(\frac{\langle-u, u\rangle}{2}\right)^{\delta}\left(\frac{-1}{\tau}\right)^{\delta}\left(\frac{\langle-v, u\rangle}{2}\right)^{\gamma}\left(\frac{-1}{\tau}\right)^{\beta} \sum_{g=1}^{m} A_{\alpha, g}^{h} \sum_{p, q, r, h, k: 2 p+r+k=\alpha, 2 q+r+h=\beta} \\
& \frac{\alpha ! \beta !}{h ! k ! r ! p ! q ! 2^{p+q}} \times S_{g}\left(\left(\tau \frac{\langle-v, v\rangle}{4 \pi \imath}\right)^{p}\left(\tau \frac{\langle-v, u\rangle}{4 \pi \imath}\right)^{r}\left(\tau \frac{\langle-u, u\rangle}{4 \pi \imath}\right)^{q}(\tau o(v))^{k}(\tau o(u))^{h}, \tau\right) \\
= & \sum_{\gamma, \delta, p, q, r, h, k} \frac{(2 \pi \imath)^{(p+k+r+q+h+\gamma+\delta)}}{\gamma ! \delta ! h ! k ! r ! p ! q !}\left(\frac{-1}{\tau}\left(\frac{\langle-u, u\rangle}{2}\right)\right)^{\delta}\left(\frac{\langle-v, u\rangle}{2}\right)^{\gamma} \times \sum_{g=1}^{m} A_{\alpha, g}^{h} S_{g}\left(\left(\tau \frac{\langle-v, v\rangle}{2}\right)^{p}(-\langle-v, u\rangle)^{r}\left(\frac{1}{\tau} \frac{\langle-u, u\rangle}{2}\right)^{q}(\tau o(v))^{k}(-o(u))^{h} ; \tau\right) \\
& \times \sum_{g=1}^{m} A_{\alpha, g}^{h} S_{g}\left(e^{2 \pi \imath\left(\frac{-1}{\tau}\langle-u, u\rangle / 2+\langle-v, u\rangle / 2+\tau\langle-v, v\rangle / 2-\langle-v, u\rangle+\frac{1}{\tau}\langle-u, u\rangle / 2+\tau o(v)-o(u)\right)} ; \tau\right)
\end{aligned}
$$




$$
\begin{aligned}
& =\sum_{g=1}^{m} A_{\alpha, g}^{h} S_{g}\left(e^{2 \pi \imath(-\langle-v, u\rangle / 2+\tau\langle-v, v\rangle / 2+\tau o(v)-o(u))} ; \tau\right) \\
& =\sum_{g=1}^{m} A_{\alpha, g}^{h} S_{g}\left(e^{2 \pi \imath(o(-u)+\langle u, v\rangle / 2)+2 \pi \imath \tau(o(v)+\langle-v, v\rangle / 2)} ; \tau\right)
\end{aligned}
$$

Namely,

$$
Z_{W_{h}}\left(v ; u ; \frac{-1}{\tau}\right)=\sum A_{\alpha, k}^{h} Z_{W_{k}}(-u ; v ; \tau)
$$

On the other hand,

$$
Z_{W_{h}}(v ; u ; \tau+1)=Z_{W_{h}}(v+u ; u ; \tau) .
$$

Therefore, for $\alpha=\left(\begin{array}{ll}a & b \\ f & d\end{array}\right)$, we have

$$
Z_{W_{h}}\left(v ; u ; \frac{a \tau+b}{f \tau+d}\right)=\sum_{k=1}^{m} A_{\alpha, k}^{h} Z_{W_{k}}(d v+b u ; f v+a u ; \tau) .
$$

\section{Q.E.D.}

Example Let $V$ be a lattice $\operatorname{VOA} V_{2 \mathbb{Z} x}$ with $\langle x, x\rangle=1$ and $W$ a module $V_{\mathbb{Z} x}$. For $z \in \mathbb{C}$, set

$$
z x=z x(-1) \mathbf{1} \in\left(V_{2 \mathbb{Z} x}\right)_{1} .
$$

Then it is easy to check by the definition of lattice VOAs that

$$
\left.\operatorname{tro}(\mathbf{1}) e^{2 \pi i z x(0)} q^{L(0)-1 / 24}\right|_{M}=\frac{1}{\eta(\tau)} \theta(\tau, z) .
$$

For $\alpha=\left(\begin{array}{cc}0 & -1 \\ 1 & 0\end{array}\right)$,

$$
\frac{1}{\eta\left(\frac{-1}{\tau}\right)} \theta\left(\frac{-1}{\tau}, z\right)=\left.\sum_{M} \lambda_{M} \operatorname{tro}(\mathbf{1}) q^{L(0)+z x(0)-\frac{1}{24}+\frac{z^{2}}{2}}\right|_{M},
$$

where $M$ runs over the following modules

$$
\left\{V_{L}, V_{x+L}, V_{\frac{1}{2} x+L}, V_{-\frac{1}{2} x+L}\right\} .
$$

Taking $\tau=i$ and several $z$, we have the known formula:

$$
\frac{1}{\eta\left(\frac{-1}{\tau}\right)} \theta\left(\frac{-1}{\tau}, z\right)=\frac{1}{\eta(\tau)} \theta(\tau, \tau z) e^{\pi i z^{2} \tau}
$$




\section{References}

[B] R. E. Borcherds, Vertex algebras, Kac-Moody algebras, and the Monster, Proc. Natl. Acad. Sci. USA 83 (1986), 3068-3071.

[CN] J. H. Conway and S. P. Norton, Monstrous moonshine, Bull. London Math. Soc. 11(1979), 308-339.

[D] C. Dong, Vertex algebras associated with even lattices, J. Algebra 160 (1993), 245-265.

[DLiM] C. Dong, H. Li and G. Mason, Modular-invariance of trace functions in orbifold theory, preprint.

[DLnM] C. Dong, Z. Lin and G. Mason, "On vertex operator algebras as $s l_{2}$-modules, in: Groups, Difference Sets, and the Monster, Proc. of a Special Research Quarter at The Ohio State University, Spring 1993, ed. by K.T. Arasu, J.F. Dillon, K. Harada, S. Sehgal and R. Solomon, Walter de Gruyter, Berlin-New York, 1996, 349-362.

[FHL] I. B. Frenkel, Y.-Z. Huang and J. Lepowsky, "On axiomatic approaches to vertex operator algebras and modules", Memoirs Amer. Math. Soc. 104, 1993.

[FLM] I. B. Frenkel, J. Lepowsky, and A. Meurman, "Vertex Operator Algebras and the Monster", Pure and Applied Math., Vol. 134, Academic Press, 1988.

[M] D. Mumford, "Tata Lectures on Theta I", Progress in Mathematics, Vol. 28, Birkhäuser, 1983.

[Z] Y. Zhu, Modular invariance of characters of vertex operator algebras, J. Amer. Math. Soc. 9 (1996), 237-302. 